\title{
Review
}

\section{Therapeutic implications of chemokine-mediated pathways in atherosclerosis: realistic perspectives and utopias}

\author{
Stavros APOSTOLAKIS, Virginia AMANATIDOU, Demetrios A SPANDIDOS* \\ Department of Clinical Virology, Faculty of Medicine, University of Crete, Voutes, Heraklion, Greece
}

Current perspectives on the pathogenesis of atherosclerosis strongly support the involvement of inflammatory mediators in the establishment and progression of atherosclerostic lesions. Chemokine-mediated mechanisms are potent regulators of such processes by orchestrating the interactions of inflammatory cellular components of the peripheral blood with cellular components of the arterial wall. The increasing evidence supporting the role of chemokine pathways in atherosclerosis renders chemokine ligands and their receptors potential therapeutic targets. In the following review, we aim to highlight the special structural and functional features of chemokines and their receptors in respect to their roles in atherosclerosis, and examine to what extent available data can be applied in disease management practices.

Keywords: chemokines; chemokine receptors; atherosclerosis; therapeutic potential

Acta Pharmacologica Sinica (2010) 31: 1103-1110; doi: 10.1038/aps.2010.131; published online 16 Aug 2010

\section{Introduction}

Atherosclerosis is an inflammatory disease involving the accumulation and activation of inflammatory cells on the vessel wall $^{[1,2]}$. In the mid-nineteenth century, Virchow ${ }^{[2]}$ proposed the importance of chronic inflammation in vascular pathology. Virchow's theory evolved over the next century into the response-to-injury hypothesis described by Ross and Glomset $^{[3]}$. The latter hypothesis suggests that the development of atherosclerotic lesions is the outcome of "some form of injury to arterial endothelium" that initiates the interaction of cellular populations of peripheral blood, with cell components of the arterial wall. Pro-inflammatory factors, chemoattractant cytokines and adhesion molecules are essential in orchestrating this process ${ }^{[1,3]}$.

\section{Vascular response to inflammatory stimulation}

Although the exact nature of "the injury to arterial endothelium" remains to be investigated, lipid accumulation is the best-supported factor in experimental atherosclerosis. Extracellular accumulation of lipids occurs in response to increased plasma lipoprotein levels ${ }^{[4,5]}$. Low-density lipoprotein (LDL) is modified rapidly in the sub-endothelial space into mini-

\footnotetext{
* To whom correspondence should be addressed.

E-mail spandidos@spandidos.gr

Received 2010-06-01 Accepted 2010-07-15
}

mally modified LDL, and subsequently into oxidized LDL (oxLDL $)^{[4,5]}$. The endothelium reacts to the "irritating" oxLDL by altering the endothelial cells' (ECs) phenotype and increasing the cell surface expression of adhesion molecules ${ }^{[4,5]}$. Circulating leukocytes that roll on the endothelium are trapped by the increased number of adhesion molecules and are forced to migrate between ECs. In the intima of the injured vessel, monocytes differentiate into macrophages; they absorb modified lipoprotein particles and become foam cells. The accumulation of foam cells in the intima results in the formation of "fatty streaks". Fatty streaks are the earliest type of atherosclerotic lesions. If the trigger factor persists, the inflammatory process continues and progresses. The initially protective inflammatory response starts to damage the arterial wall. Dysfunctional ECs and activated leukocytes release cytokines, chemokines and growth factors, and promote the migration of smooth muscle cells (SMCs) into the intima of the arterial wall, leading to more complicated lesions. At this stage, the lesion contains multiple layers of SMCs, connective tissue, macrophages and $\mathrm{T}_{\text {cells }}{ }^{[4,5]}$. The lesions are affected by pre-disposing factors, resulting in the lesions evolving further while remodelling of the vessel wall occurs in a final effort to retain sufficient flow. The final stage of atheromas or advanced lesions is susceptible to erosion and rapture, the pathophysiological background of acute ischemic events ${ }^{[4,5]}$. 


\section{Chemokine structural and functional characteristics}

Chemokines are low molecular weight chemoattractant cytokines that serve as significant regulatory proteins in leukocyte trafficking and activation. They are classified into four sub-families based on the number and structural arrangement of conserved cysteine residues within their amino-terminal polypeptide sequence (C, CC, CXC, and CX3C). CXC chemokines have a single amino acid separating the two amino-terminal cysteine residues of the protein, while CC chemokines lack this type of amino acid sequence. CX3CL1 is the only member of the CX3C sub-family to have three amino acids separating the two amino-terminal cysteine residues. Finally, lymphotactine (XCL1) and single C motif chemokine 1 (SCM1 or XCL2) are the only currently known members of the $C$ sub-family and lack two of the four conserved cysteines in the mature $\operatorname{protein}^{[6]}$.

Chemokines induce cell activation by binding to specific seven-transmembrane G-protein coupled cell-surface receptors on target cells. A total of six human CXC chemokine receptors, 10 human $\mathrm{CC}$ chemokine receptors, and a single receptor for each of the $\mathrm{CX} 3 \mathrm{C}$ and $\mathrm{C}$ chemokine sub-families have been identified thus far. An unusual characteristic of the majority of chemokine receptors is their high affinity for multiple ligands. The latter characteristic implies that the same ligand can cause a variety of biological effects depending on the type of chemokine receptor most profoundly expressed by the target cells. Chemokines interact with their receptors on the cell surface leading to the generation of an intracellular signal via the G-protein complex, and subsequently to cell chemotaxis towards the source of the chemokine ${ }^{[6]}$. Chemokines promote chemotaxis through the development of a chemotactic gradient that mobilises the inflammatory cell towards an area of increased chemokine concentration. In vivo, the chemotactic gradient may be generated by the binding of chemokines to basement membrane proteins. This gradient aids in transferring cells towards the site of inflammation and retaining them once they have reached the area of interest ${ }^{[6,7]}$. Chemokine biology is nonetheless far more complex than a simple ligandreceptor interaction. Several studies have suggested that chemokines can dimerize and that their receptors are found as dimers and/or higher order oligomers at the cell surface. Moreover, functional studies indicate considerable synergy between chemokines, and chemokine heteromerization has more recently emerged as an additional regulatory mechanism. Synergy augments leukocyte chemotaxis and activation $^{[8,9]}$. For example, the inflammatory chemokine CXCL8 can promote responses induced by CCL2 and CXCL12, which act on CCR1 and CXCR4, respectively, but not those induced by CCL21, which triggers responses through CCR7 ${ }^{[10]}$. Similarly, mutual modulation of CXCL8 and CXCL4 activities has been suggested, as well as enhanced monocyte arrest resulting from CCL5-CXCL4 interactions ${ }^{[11]}$. In vivo data also indicate that, for some ligands, oligomer formation, such as CCL2, CCL4, CCL5, and CXCL10, enhances leukocyte recruitment ${ }^{[12]}$. Finally, the chemokine system is highly influenced by the microenvironmental context and regulation of the chemokine system occurs not only at the level of agonist production, but also at the level of chemokine receptor expression ${ }^{[13]}$.

The response-to-injury hypothesis increased the interest of the role of chemokines in atherosclerosis since they were ideal candidates for the regulation of essential aspects of atherogenesis, such as the recruitment of inflammatory cells onto the vessel wall and the proliferation of SMCs in atherosclerotic plaques $^{[6]}$. This vital involvement of chemokines in the establishment and progression of athrosclerosis created the impression that chemokines and their receptors may provide novel targets for therapeutic interventions in atherosclerosis-related diseases, such as coronary artery disease (CAD), peripheral artery disease (PAD) and cerebrovascular disease. The present review attempts to provide recent evidence supporting the role of chemokines in atherosclerosis and examines how the information obtained may be applied in therapeutic practices. We restricted our assessment to selected chemokine/chemokine receptor systems. It is quite likely, however, that other aspects related to chemokines may well play an important role in atherogenesis.

\section{Chemokine-mediated pathways in atherosclerosis}

Each stage of atherosclerosis is characterised by different cellular interactions and subsequently regulated by different cytokines, growth factors and adhesion molecules ${ }^{[14]}$. The most pronounced event of the early stages of atherogenesis is chemotaxis and migration of the rolling monocytes in the intima of the injured vessel. In this stage, oxLDL induces the expression of CCL2 and CX3CL1, by SMCs and ECs ${ }^{[15,16]}$. The interaction of CX3CL1 and CCL5, with their receptors CX3CR1 and CCR1, respectively, is currently considered to be an early pathway leading to the firm adhesion of rolling monocytes to stimulated endothelium ${ }^{[15-17]}$. CX3CL1 as a structurally unique chemokine acts both as a chemoattractant and as an efficient adhesion molecule through a non-integrindependent mechanism ${ }^{[6]}$. Soluble CCL2, secreted by ECs and SMCs, induces structural changes in the cytoskeleton of CCR2expressing adherent monocytes, potentiating transendothelial migration $^{[15-17]}$. Concurrently, CXC chemokines induced by interferon gamma, such as CXCL10, CXCL9, and CXCL11 expressed predominantly by ECs interact with CXCR3expressing $\mathrm{T}$ cells, inducing their accumulation and migration, and subsequently increasing the vascular inflammatory response $^{[15-17]}$. Recruitment of neutrophils and vascular progenitor cells in atherosclerotic lesions is controlled by CXCR2 and CXCR4, and their ligands CXCL8 and CXCL1. CXCL8 is highly expressed by lesion macrophages, as well as by ECs and SMCs. CXCL8, although mainly a granulocyte chemoattractant, also induces the firm adhesion of CXCR2-expressing monocytes to the endothelium under physiological flow conditions $^{[18]}$. As in the case of CCL5, and CX3CL1, CXCL8 promotes the firm adhesion of rolling monocytes in the early stages of atherogenesis ${ }^{[15]}$.

Therefore, in the course of atherosclerosis, chemokines form a complicated network by promoting specific cellular interactions. Different chemokines promote different pathways. 
Moreover, the interaction of the same chemokine ligand with different receptors results in a different outcome. This phenomenally crucial implication of chemokines in atherosclerosis generates two clinically relevant questions: can chemokineinduced pathways be blocked? And most importantly: are chemokine pathways realistic therapeutic targets?

\section{CCL2 and CC receptor 2}

\section{The facts}

CCL2 was until recently the leading chemokine used in experimental atherosclerosis. It is the prototype molecule of the CC class and a strong chemoattractant for monocytes. The presence of CCL2 in atherosclerotic lesions was first demonstrated in 1991 by in situ hybridisation and has since been confirmed by a number of studies and multiple experimental settings ${ }^{[19]}$. CCL2 mRNA has been detected in ECs, macrophages and vascular SMCs in atherosclerotic arteries ${ }^{[20-22]}$. Various experimental models of atherosclerosis, including LDL receptor and apolipoprotein E knockout (LDLr-/-, ApoE-/-) mice, have been used to confirm the role of CCL2 or its receptor, CCR2, in atherosclerosis. Gu et al reported less lipid deposition and fewer macrophages in the aortic walls of LDLr-/- mice that lacked the CCL2 encoding gene ${ }^{[23]}$. Similarly, Boring et al, using ApoE-/- knockout mice, observed an overall decrease in atherosclerotic lesions in mice deficient of the CCL2 receptor $^{[24]}$. Aiello et al demonstrated that the overexpression of CCL2 in the bone marrow-derived cells of ApoE-/- mice resulted in increased lesion formation as well as an increased accumulation of oxidized lipids and macrophages ${ }^{[25]}$. In a study by Roque et al, mice deficient in CCR2 significantly reduced intimal hyperplasia following injury to the femoral artery $^{[26]}$. Experimental blocking of the CCL2/CCR2 pathway in atherosclerotic models also resulted in a significant reduction of the atherosclerotic burden. Ni et al demonstrated a new strategy for anti-CCL2 gene therapy to treat atherosclerosis by transfecting an N-terminal deletion mutant of the human CCL2 gene into the skeletal muscle in ApoE-/- mice. This strategy effectively blocked CCL2 activity and inhibited the formation of atherosclerotic lesions. Furthermore, it increased the lesional extracellular matrix content. These authors concluded that anti-CCL2 gene therapy may serve not only to reduce atherogenesis but also to stabilize vulnerable atheromatous plaques. Thus, it was proposed that this strategy is a potentially useful and plausible form of gene therapy against atherosclerosis in humans ${ }^{[27]}$. Similarly, Inoue et al reported that blockade of CCL2 by transfecting an N-terminal deletion mutant of the CCL2 gene limited the progression of pre-existing atherosclerotic lesions in the aortic root in hypercholesterolemic mice, changing the lesion composition into a more stable phenotype ${ }^{[28]}$. Usui et al demonstrated that transfection of an N-terminal deletion mutant of the CCL2 gene into skeletal muscles suppressed monocyte infiltration/activation in the injured site and markedly inhibited restenotic changes after balloon injury of the carotid artery in rats and monkeys. This strategy also suppressed the local production of CCL2 and inflammatory cytokines. The authors concluded that monocyte infiltration and activation mediated by CCL2 are essential in the development of restenotic changes after balloon injury and suggested this strategy as a potential form of gene therapy against human restenosis ${ }^{[29]}$. Clinical studies also assessed the importance of CCL2 as a marker of atherosclerosis and supported its use as a therapeutic target. Hoogeveen et al evaluated the relationship between CCL2 plasma levels and peripheral arterial disease (PAD) or coronary heart disease (CHD). They reported a significant correlation of CCL2 plasma levels with PAD, independent of traditional CHD risk factors. Moreover, CHD risk was significantly correlated to the CCL2 level independent of other cardiovascular risk factors. The authors concluded that CCL2 is associated with atherosclerotic disease in two vascular beds, and suggested that CCL2 may be a novel therapeutic target for atherosclerosis $^{[30]}$. van Wijk DF et al assessed prospectively the relationship between CCL2 single-nucleotide polymorphisms (SNPs), CCL2 serum concentrations, and the risk of future CAD. The authors failed to establish an association between CCL2 SNPs and serum concentrations, or a significant association between CCL2 SNPs, CCL2 serum levels and the risk of future CAD. Thus, these authors concluded that their results do not support that CCL2 plays a role in the pathogenesis of CAD ${ }^{[31]}$. Finally, in the largest available case-control study, de Lemos et al examined whether CCL2 adds prognostic value to standard risk assessment tools and biomarkers following acute coronary syndromes (ACS). The authors reported that the rates of death and the composite end points of death or myocardial infarction (MI) increased across baseline quartiles of CCL2 and among patients with CCL2 greater than the pre-specified threshold. Following adjustment for standard risk predictors and levels of C-reactive protein and B-type natriuretic peptide, CCL2 remained independently associated with mortality. The authors concluded that CCL2 provides independent prognostic value in the acute and chronic phases after ACS and merits further evaluation as a prognostic marker and potential therapeutic target $^{[32]}$.

\section{The present and the future}

Although promising results from animal models were noted, the blockade of the CCL2/CCR2 pathway has yet to be examined as a potential therapeutic target in human atherosclerosis. In the last decade, the literature has largely investigated the CCL2/CCR2 pathway as a potential marker rather than a therapeutic target. Case-control studies resulted in controversial results and those studies that reported the potential importance of the CCL2/CCR2 pathway as a therapeutic target have only indirectly assessed the issue. Studies should therefore be conducted that will evaluate the CCL2/CCR2 blockade as a potential therapeutic target in human atherosclerosis.

\section{CCL5 and CC receptors 1/5}

\section{The facts}

CCL5 is expressed by T lymphocytes, macrophages, myofibroblasts and endothelial cells in atherosclerosis. Platelets are also an important source ${ }^{[33,34]}$. CCL5 binds to CCR1, CCR3, 
and $\mathrm{CCR} 5^{[33]}$, and induces the adhesion and migration of monocytes and $\mathrm{T}$ lymphocytes in an integrin-dependent manner ${ }^{[35,36]}$. The interaction of CCL5, with its receptor CCR1, is currently considered to be an early pathway leading to the firm adhesion of rolling monocytes to stimulated endothelium $^{[15-17]}$. Pattison et al investigated the expression of CCL5 using in situ hybridisation and immunohistochemistry in the coronary arteries of patients undergoing accelerated atherosclerosis compared to normal coronary arteries ${ }^{[37]}$. They demonstrated that CCL5 mRNA and protein were detected in the lymphocytes, macrophages, myofibroblasts and ECs of atherosclerotic arteries but not in normal vessels. The authors concluded that CCL5 may be a pivotal mediator of the cellular infiltrate noted in graft atherosclerosis. CCL5 has also been assessed as an important mediator of platelet-EC interaction. Huo et al demonstrated in apolipoprotein-E-deficient mice that platelets deliver CCL5 to the monocyte surface and endothelium-promoting leukocyte adhesion to the vascular wall ${ }^{[38]}$. von Hundelshausen et al reported that the combination of CCL5 and CXCL4 promotes monocyte adhesion to activated human umbilical vein ECs under flow conditions to a greater extent than each of the chemokines alone ${ }^{[39]}$. The authors proposed that heterophilic interactions with CXCL4 require structural motifs that are important in CCL5 oligomerization and amplify CCL5-triggered effects on monocyte adhesion. Koenen et al determined structural features of CCL5-CXCL4 heteromers and designed stable peptide inhibitors that specifically disrupt pro-inflammatory CCL5-CXCL4 interactions. The authors concluded that their results support the in vivo relevance of chemokine heteromers and show the potential of targeting heteromer formation to achieve therapeutic effects ${ }^{[40]}$. In a completely different setting, Veillard et al reported that blocking in vivo CCL5-mediated signalling using the CC chemokine antagonist Met-RANTES, reduced the progression of atherosclerosis in hypercholesterolemic animal models, indicating that the blockade of chemokine receptor/ligand interactions may become a novel therapeutic target ${ }^{[41]}$. Braunersreuther et al investigated the effect of chemokine CCL5/RANTES antagonism in a mouse model of ischemia and reperfusion. The authors reported that intraperitoneal injection of the CCL5 antagonist [(44)AANA(47)]-RANTES, 5 min prior to reperfusion, reduced infarct size as well as Troponin I serum levels and reduced inflammatory response. The authors concluded that CCL5 exerts cardioprotective effects during early myocardial reperfusion and proposed that blocking chemokine receptor/ligand interactions may become a novel therapeutic strategy to reduce reperfusion injuries in patients during $\mathrm{ACS}^{[42]}$.

\section{The present and the future}

Promising data exist to support the therapeutic potential of the blockade of CCL5-mediated pathways. Met-RANTES has previoiusly been investigated; however, it has never been nor is it likely to be tested in human atherosclerosis. Met-RANTES reduces diet-induced atherosclerosis. However, CCL5 antagonism may not be therapeutically feasible, as suggested by studies using CCL5-deficient mice, the former of which imply that the direct CCL5 blockade compromised systemic immune responses, delayed macrophage-mediated viral clearance and impaired normal T-cell functions. Peptide inhibitors that specifically disrupt pro-inflammatory CCL5-CXCL4 interactions are novel and probably more realistic therapeutic mediators; however, data related to these interactions are currently limited.

\section{CXCL8 and CXC receptors $1 / 2$}

\section{The facts}

CXCL8 is the prototypical chemokine of the CXC subfamily. CXCL8 is actively secreted as a result of a variety of cellular stimuli. Although all nucleated cells are able to generate CXCL8, its principal cellular sources are monocytes and macrophages. Monocytes and neutrophils are the main targets of CXCL8 ${ }^{[43]}$. The biological effects of CXCL8 are mediated through the binding of CXCL8 to two cell surface receptors, CXCR1 and CXCR2. These G-protein-coupled receptors share considerable structural similarity and induce a nearly identical range of biological activities. Since the discovery of CXCL8, in 1987, information pertaining to its role in leukocyte infiltration has rapidly advanced and focused significantly on its implications in vascular pathology ${ }^{[43]}$. Apostolopoulos et al demonstrated the expression of CXCL8 in human atherosclerotic plaques by in situ hybridisation ${ }^{[44]}$. CXCL8 has been shown to contribute in SMC proliferation and migration ${ }^{[45]}$ and, although CXCL8 has been thought to act predominantly on neutrophils, Gerszten et al demonstrated that it induced firm adhesion of rolling monocytes to ECs expressing E-selec$\operatorname{tin}^{[46]}$. In a more recent study, Huo et al, using isolated carotid artery from ApoE-/- mice, demonstrated that CXCL1, but not CCL2, plays a role in monocyte arrest on the endothelium of atherosclerosis-prone vessels ${ }^{[47]}$. Boyle $e t$ al evaluated the effect of direct CXCL8 inhibition on the degree of myocardial injury encountered during reperfusion. Moreover, these authors demonstrated that the neutralisation of CXCL8 significantly reduced the degree of necrosis in a rabbit model of myocardial ischaemia reperfusion injury ${ }^{[48]}$. Boisvert et al used LDLr-/- mice which were irradiated and repopulated with bone marrow cells, but lacked the murine homologue of CXCR2 ${ }^{[49]}$. The authors reported that CXCR2-deficient animals had fewer extensive lesions and fewer macrophages than those mice receiving bone marrow cells expressing the receptor. Schwartz et al reported that CXCL1, another ligand of CXCR2, may similarly contribute to the adhesion of monocytes to minimally modified LDL-stimulated $\mathrm{ECs}^{[50]}$. Weber et al further proposed that macrophage migration inhibitory factor (MIF) is another potential CXCR2 ligand. The authors demonstrated that MIF possesses a pseudo-(E)LR motif that enables MIF to act as a non-canonical CXCR2 ligand, and concluded that this structural resemblance may be the background of pro-inflammatory MIF/CXCR2 interactions ${ }^{[51]}$. In support of this hypothesis, the deletion of CXCL1 in LDLr-/- mice reduces atherosclerosis to a lesser extent than bone marrow CXCR2 deficiency in LDLr-/- mice ${ }^{[14]}$. Thus, CXCR2 appears to be the key regulator in the ligand/receptor pathway and a 
more promising therapeutic target than the direct blockade of CXCL8.

\section{The present and the future}

Sufficient data exist that support the role of CXCL8 in atherosclerosis, the therapeutic potential of CXCR2 and to a lesser extent, the CXCL8 blockade. However, these results have yet to be investigated in human atherosclerosis in clinical settings.

\section{CX3CL1 and CX3C receptor 1}

\section{The facts}

CXCL1 is the only member of the CX3C chemokine subfamily. It is membrane-bound and in soluble form. Soluble CX3CL1 is released, presumably by proteolysis, at the membrane-proximal region by a TNF-converting enzyme. Soluble CX3CL1 is an efficient chemoattractant for monocytes and natural killer cells. The receptor of CX3CL1, CX3CR1, is a seven-transmembrane domain G protein-coupled receptor, and CX3CL1 binds to it with high affinity, activating intracellular signalling and directly mediating monocyte adhesion ${ }^{[6]}$. Through its unique structural and functional characteristics, CX3CL1 exhibits properties of both chemokine and adhesion molecules ${ }^{[6]}$. The CX3CL1/CX3CR1 pathway, has been shown to play a role in atherogenesis ${ }^{[6]}$ in different settings. Immunochemical studies have confirmed the expression of this pathway in monocytes/ macrophages, ECs and SMCs within human atherosclerotic coronary arteries ${ }^{[52]}$. Lucas et al reported that SMCs in the neointima of human atherosclerotic plaques express CX3CR1. The authors further demonstrated that primary cultured human coronary artery SMCs migrate toward CX3CL1, suggesting that CX3CR1 also induces the migration of SMCs to atherosclerotic lesions ${ }^{[53]}$. Yang et al demonstrated that vascular ECs expressed CX3CL1 and its receptor CX3CR1. Soluble CX3CL1 is released from ECs following hypoxia/ reoxygenation and acts through CX3CR1 on ECs to increase ICAM-1 expression and promote neutrophil adhesion ${ }^{[54]}$. In a similar setting, Schulz et al reported that CX3CL1 expressed by inflamed ECs triggers P-selectin exposure on adherent platelets, which initiates the local accumulation of leukocytes under arterial shear ${ }^{[5]}$. Barlic et al demonstrated that CX3CR1 and CX3CL1 mediated the heterotypic anchorage of foam cells to coronary artery SMCs and suggested that the CX3CL1/ CX3CL1 pathway is likely a pro-inflammatory target for therapeutic intervention in atherosclerotic cardiovascular disease ${ }^{[56]}$. White et al demonstrated that CX3CL1 possesses anti-apoptotic and proliferating properties on primary human SMCs. The authors concluded that the latter CX3CL1-mediated effects play a significant role in vascular pathologies including atherosclerosis, restenosis, and transplant-accelerated arteriosclerosis, where the balance of SMC proliferation and apoptosis critically determines both plaque stability and vessel stenosis $^{[57]}$. In a recent study of ours, higher rates of CX3CR1-positive monocytes were observed in CAD patients as compared to subjects with normal coronary angiography. Furthermore, we demonstrated in vitro that a pre-inflammatory environment induced by INF-gamma enhances the rate and fluorescence intensity of CX3CR1-positive THP-1 monocytes, indicating that regulation of the chemokine system occurs not only at the level of agonist production, but also at the level of CX3CR1 receptor expression ${ }^{[58]}$. Studies have also implicated CX3CL1 in platelet stimulation and activation. Activated platelets have been shown to exacerbate atherosclerosis in murine models of atherogenesis. Schafer et al demonstrated that platelets from rats, pre-incubated with CX3CL1, increased the P-selectin surface expression. In addition, pre-incubation with CX3CL1 enhanced platelet adhesion to collagen and fibrinogen ${ }^{[59]}$. Combadiere et al indirectly examined the therapeutic potential of CX3CL1 in double knockout mice (ApoE-/-, CX3CR1-/-). The results of these authors showed a significant decrease in lesion size in animals lacking the CX3CL1-CX3CR1 signalling mechanism $^{[60]}$. Saederup et al reported in a triple knockout animal model (CX3CR1-/-, CCR2-/-, and ApoE-/-) that the deletion of CX3CL1 in CCR2-/- mice markedly reduced macrophage accumulation in the arterial wall. The authors concluded that successful therapeutic strategies may need to target multiple chemokines or chemokine receptors ${ }^{[61]}$. Landsman et al reported that the absence of either CX3CL1 or CX3CR1 results in a significant reduction of Gr1(low) blood monocyte levels under both steady-state and inflammatory conditions. These authors further showed that CX3CL1 specifically rescued cultured human monocytes from induced cell death and concluded that CX3CL1/CX3CR1 interactions confer an essential survival signal to monocytes and/ or foam cells ${ }^{[2]}$. Using a phage display strategy, Dorgham et al engineered a hCX3CL1 analogue (named F1) with a modified $\mathrm{N}$ terminus and a similar affinity for hCX3CR1 as the native CX3CL1. F1 potently inhibited the CX3CL1-induced calcium flux and chemotaxis in CX3CR1-expressing primary cells of human and murine origin. It also efficiently inhibited CX3CL1-mediated cell adhesion. Finally, in a non-infectious murine model of peritonitis, F1 strongly inhibited macrophage accumulation. The authors suggested that this molecule be used as a lead compound for the development of a novel class of anti-inflammatory substances that act by inhibiting CX3CR1 ${ }^{[63]}$.

\section{The present and the future}

The CX3CL1/CX3CR1 pathway is the most promising chemokine-related therapeutic target in atherosclerosis. The unique structural characteristics of CX3CL1 and the noteworthy yet high quality evidence of its role in monocyte accumulation migration and survival in human atherosclerosis suggest that this pathway deserves further evaluation. The recent report on the development of the synthetic CX3CL1-blocking analogue provides the means of a direct evaluation of the CX3CR1 blockade in experimental atherosclerosis. Therefore, these means should be further studied.

\section{Discussion}

It is well known that CCL2/CCR2, CXCL8/CXCR2, CCL5/ CCR1, and CX3CL1/CX3CR1 are key mediators of atherogenesis, which is clearly suggested by high-quality evidence 
provided by in vitro studies, studies in animal models of accelerated atherosclerosis, case-control studies and studies in genetic epidemiology ${ }^{[6]}$. However, the therapeutic potential of blocking chemokine pathways in the field of atherosclerotic disease remains to be elucidated. Various chemokine receptor antagonists are currently under clinical evaluation as therapeutic targets in other inflammatory diseases; the efficacy and safety of CCR1 antagonists has been evaluated for the treatment of rheumatoid arthritis ${ }^{[64]}$. A monoclonal antibody blocking the binding of CCL2 to CCR2 was tested for the treatment of rheumatoid arthritis ${ }^{[65]}$. CCR5 antagonists are currently in advanced stages of clinical development as adjuvant treatments for AIDS ${ }^{[6,67]}$. Although promising results in other disease states have been observed, we are unaware of any clinical studies designed or currently being conducted to examine the therapeutic efficacy of chemokine blocking in atherosclerotic disease. All investigators agree that the precise mechanism of the chemokine pathways involved in the establishment and progression of atherosclerosis has yet to be elucidated and much more information is required before chemokine-based therapies can be tested in a clinical setting. Moreover, therapeutic approaches to cardiovascular disease may affect a considerable amount of patients and, thus, should be evaluated in large-scale randomised controlled trials with specific 'hard' endpoints. However, randomised controlled trials require complicated multi-centre design and significant financial resources. As in any immune-modulating therapeutic approach, several drawbacks need to be considered before chemokine blockade is tested in any clinical setting. First of all, atherosclerosis is a chronic condition; thus, long-term immune inhibition must be applied. This raises tolerability and safety issues for potential therapeutic agents that block a non-disease-specific chemotactic pathway. Secondly, chemokines are important mediators of various aspects of immune response. Therefore, the complete blockade of a chemokine signalling pathway may not be desirable. Techniques need to be applied to ensure that chemokine blocking occurs in specific locations and tissues, resulting in a beneficial net effect. Finally, pharmacokinetic and pharmacoeconimical issues should be resolved since peptide analogues can be problematic and expensive especially if it is necessary for these analogues to be administered for long-term treatment. An appealing area of investigation for immunosuppressive therapies is restenosis after percutaneous coronary intervention, in which chemokine blockers may be tested as potential agents for stent elution. This latter approach ensures better targeting and fewer side effects than systemic treatment. Moreover, if chemokine antagonism was restricted to a limited area, multiple receptor-blockade could be applied without compromising systemic immune response. Since extensive cross talk between chemokine ligands and receptors has been demonstrated, multiple receptor blockade might diminish local inflammation and attenuate restenosis. Thus, targeting chemokine pathways in an effort to reduce the rate of restenosis is a challenging field of investigation. Currently, however, no such data exist.

In conclusion, a significant amount of evidence can be found supporting the therapeutic potential of certain chemokine/ chemokine receptor blockade. However, numerous issues should be resolved before chemokine-based therapeutic approaches can be used in clinical settings.

\section{References}

1 Ross R. Atherosclerosis - an inflammatory disease. N Engl J Med 1999; 340: 115-26.

2 Virchow R. Phlogose und thrombose in gefassystem. In: Gesammelte Abhandlungen zur Wissenschaftlichen Medicin. Frankfurt, Germany: Staatsdruckerei, 1856. p458-68.

3 Ross R, Glomset JA. Atherosclerosis and the arterial smooth muscle cell. Science 1973; 180: 1332-6.

4 Libby P. Inflammation in atherosclerosis. Nature 2002; 420: 868-74.

5 Luster AD. Chemokines - chemotactic cytokines that mediate inflammation. N Engl J Med 1998; 338: 436-45.

6 Apostolakis S, Papadakis EG, Krambovitis E, Spandidos DA. Chemokines in vascular pathology (review). Int J Mol Med 2006; 17: 691-701.

7 Gimbrone MA Jr, Obin MS, Brock AF, Luis EA, Hass PE, Hebert CA, et al. Endothelial interleukin-8: a novel inhibitor of leukocyte-endothelial interactions. Science 1989; 246: 1601-3.

8 Thelen M, Muñoz LM, Rodríguez-Frade JM, Mellado M. Chemokine receptor oligomerization: functional considerations. Curr Opin Pharmacol 2010; 10: 38-43.

9 von Hundelshausen P, Koenen RR, Sack M, Mause SF, Adriaens W, Proudfoot AE, et al. Heterophilic interactions of platelet factor 4 and RANTES promote monocyte arrest on endothelium. Blood 2005; 105: 924-30.

10 Gouwy M, Struyf S, Catusse J, Proost P, Van DJ. Synergy between proinflammatory ligands of $G$ protein-coupled receptors in neutrophil activation and migration. J Leukoc Biol 2004; 76: 185-94.

11 Koenen RR, von Hundelshausen P, Nesmelova IV, Zernecke A, Liehn EA, Sarabi A, et al. Disrupting functional interactions between platelet chemokines inhibits atherosclerosis in hyperlipidemic mice. Nat Med 2009; 15: 97-103.

12 Proudfoot AE, Handel TM, Johnson Z, Lau EK, LiWang P, Clark-Lewis I, et al. Glycosaminoglycan binding and oligomerization are essential for the in vivo activity of certain chemokines. Proc Natl Acad Sci USA 2003; 100: 1885-90.

13 Locati M, Riboldi E, Otero K, Martinez FO, Riva F, Perrier P, et al. Regulation of the chemokine system at the level of chemokine receptor expression and signaling activity. Immunobiology 2001; 204: 536-42.

14 Zernecke A, Shagdarsuren E, Weber C. Chemokines in atherosclerosis: an update. Arterioscler Thromb Vasc Biol 2008; 28: $1897-$ 908.

15 Weber C, Schober A, Zernecke A. Chemokines: key regulators of mononuclear cell recruitment in atherosclerotic vascular disease. Arterioscler Thromb Vasc Biol 2004; 24: 1997-2008.

16 Barlic J, Murphy PM. Chemokine regulation of atherosclerosis. J Leukoc Biol 2007; 82: 226-36.

17 Weber KS, von Hundelshausen P, Clark-Lewis I, Weber PC, Weber C. Differential immobilization and hierarchical involvement of chemokines in monocyte arrest and transmigration on inflamed endothelium in shear flow. Eur J Immunol 1999; 29: 700-12.

18 Mehra VC, Ramgolam VS, Bender JR. Cytokines and cardiovascular disease. J Leukoc Biol 2005; 78: 805-18.

19 Yla-Herttuala S, Lipton BA, Rosenfeld ME, Sarkioja T, Yoshimura T, Leonard EJ, et al. Expression of monocyte chemoattractant protein 1 in macrophage-rich areas of human and rabbit atherosclerotic lesions. 
Proc Natl Acad Sci USA 1991; 88: 5252-6.

20 Takeya M, Yoshimura T, Leonard EJ, Takahashi K. Detection of monocyte chemoattractant protein-1 in human atherosclerotic lesions by an anti-monocyte chemoattractant protein-1 monoclonal antibody. Hum Pathol 1993; 24: 534-9.

21 Nelken NA, Coughlin SR, Gordon D, Wilcox JN. Monocyte chemoattractant protein-1 in human atheromatous plaques. J Clin Invest 1991; 88: 1121-7.

22 Seino Y, Ikeda U, Takahashi M, Hojo Y, Irokawa M, Kasahara T, et al. Expression of monocyte chemoattractant protein-1 in vascular tissue. Cytokine 1995; 7: 575-9.

23 Gu L, Okada Y, Clinton SK, Gerard C, Sukhova GK, Libby P, et al. Absence of monocyte chemoattractant protein-1 reduces atherosclerosis in low density lipoprotein receptor-deficient mice. Mol Cell 1998; 2: 275-81.

24 Boring L, Gosling J, Cleary M, Charo IF. Decreased lesion formation in CCR2-/- mice reveals a role for chemokines in the initiation of atherosclerosis. Nature 1998; 394: 894-897.

25 Aiello RJ, Bourassa PA, Lindsey S, Weng W, Natoli E, Rollins BJ, et al. Monocyte chemoattractant protein-1 accelerates atherosclerosis in apolipoprotein E-deficient mice. Arterioscler Thromb Vasc Biol 1999; 19: 1518-25.

26 Roque M, Kim WJ, Gazdoin M, Malik A, Reis ED, Fallon JT, et al. CCR2 deficiency decreases intimal hyperplasia after arterial injury. Arterioscler Thromb Vasc Biol 2002; 22: 554-9.

27 Ni W, Egashira K, Kitamoto S, Kataoka C, Koyanagi M, Inoue S, et al. New anti-monocyte chemoattractant protein-1 gene therapy attenuates atherosclerosis in apolipoprotein E-knockout mice. Circulation 2001; 103: 2096-101.

28 Inoue S, Egashira K, Ni W, Kitamoto S, Usui M, Otani K, et al. Antimonocyte chemoattractant protein-1 gene therapy limits progression and destabilization of established atherosclerosis in apolipoprotein E-knockout mice. Circulation 2002; 106: 2700-6.

29 Usui M, Egashira K, Ohtani K, Kataoka C, Ishibashi M, Hiasa K, et al. Anti-monocyte chemoattractant protein-1 gene therapy inhibits restenotic changes (neointimal hyperplasia) after balloon injury in rats and monkeys. FASEB J 2002; 16: 1838-40.

30 Hoogeveen RC, Morrison A, Boerwinkle E, Miles JS, Rhodes CE, Sharrett AR, et al. Plasma MCP-1 level and risk for peripheral arterial disease and incident coronary heart disease: Atherosclerosis Risk in Communities study. Atherosclerosis 2005; 183: 301-7.

31 van Wijk DF, van Leuven SI, Sandhu MS, Tanck MW, Hutten BA, Wareham NJ, et al. Chemokine ligand 2 genetic variants, serum monocyte chemoattractant protein-1 levels, and the risk of coronary artery disease. Arterioscler Thromb Vasc Biol 2010; 30: 1460-6.

32 de Lemos JA, Morrow DA, Blazing MA, Jarolim P, Wiviott SD, Sabatine MS, et al. Serial measurement of monocyte chemoattractant protein-1 after acute coronary syndromes: results from the A to $Z$ trial. J Am Coll Cardiol 2007; 50: 2117-24.

33 Kameyoshi Y, Dorschner A, Mallet Al, Christophers E, Schroder JM. Cytokine RANTES released by thrombin-stimulated platelets is a potent attractant for human eosinophils. J Exp Med 1992; 176: 587 92.

34 von Hundelshausen P, Weber C. Platelets as immune cells: bridging inflammation and cardiovascular disease. Circ Res 2007; 100: 27 40.

35 Gilat D, Hershkoviz R, Mekori YA, Vlodavsky I, Lider O. Regulation of adhesion of CD4+ T lymphocytes to intact or heparinase-treated subendothelial extracellular matrix by diffusible or anchored RANTES and MIP-1 beta. J Immunol 1994; 153: 4899-906.

36 von Hundelshausen P, Weber KS, Huo Y, Proudfoot AE, Nelson PJ, Ley
$\mathrm{K}$, et al. RANTES deposition by platelets triggers monocyte arrest on inflamed and atherosclerotic endothelium. Circulation 2001; 103: 1772-7.

37 Pattison JM, Nelson PJ, Huie P, Sibley RK, Krensky AM. RANTES chemokine expression in transplant-associated accelerated atherosclerosis. J Heart Lung Transplant 1996; 15: 1194-9.

38 Huo Y, Schober A, Forlow SB, Smith DF, Hyman MC, Jung S, et al. Circulating activated platelets exacerbate atherosclerosis in mice deficient in apolipoprotein E. Nat Med 2003; 9: 61-7.

39 von Hundelshausen P, Koenen RR, Sack M, Mause SF, Adriaens W, Proudfoot $\mathrm{AE}$, et al. Heterophilic interactions of platelet factor 4 and RANTES promote monocyte arrest on endothelium. Blood 2005; 105: 924-30.

40 Koenen RR, von Hundelshausen P, Nesmelova IV, Zernecke A, Liehn EA, Sarabi A, et al. Disrupting functional interactions between platelet chemokines inhibits atherosclerosis in hyperlipidemic mice. Nat Med 2009; 15: 97-103.

41 Veillard NR, Kwak B, Pelli G, Mulhaupt F, James RW, Proudfoot AE, et al. Antagonism of RANTES receptors reduces atherosclerotic plaque formation in mice. Circ Res 2004; 94: 253-61.

42 Braunersreuther V, Pellieux C, Pelli G, Burger F, Steffens S, Montessuit $\mathrm{C}$, et al. Chemokine CCL5/RANTES inhibition reduces myocardial reperfusion injury in atherosclerotic mice. J Mol Cell Cardiol 2010; 48: 789-98.

43 Apostolakis S, Vogiatzi K, Amanatidou V, Spandidos DA. Interleukin 8 and cardiovascular disease. Cardiovasc Res 2009; 84: 353-60.

44 Apostolopoulos J, Davenport P, Tipping PG. Interleukin-8 production by macrophages from atheromatous plaques. Arterioscler Thromb Vasc Biol 1996; 16: 1007-12.

45 Yue TL, Wang X, Sung CP, Olson B, McKenna PJ, Gu JL, et al. Interleukin-8. A mitogen and chemoattractant for vascular smooth muscle cells. Circ Res 1994; 75: 1-7.

46 Gerszten RE, Garcia-Zepeda EA, Lim YC, Yoshida M, Ding HA, Gimbrone MA Jr, et al. MCP-1 and IL-8 trigger firm adhesion of monocytes to vascular endothelium under flow conditions. Nature 1999; 398: 718-23.

47 Huo Y, Weber C, Forlow SB, Sperandio M, Thatte J, Mack M, et al. The chemokine $\mathrm{KC}$, but not monocyte chemoattractant protein-1, triggers monocyte arrest on early atherosclerotic endothelium. J Clin Invest 2001; 108: 1307-14.

48 Boyle EM Jr, Kovacich JC, Hebert CA, Canty TG Jr, Chi E, Morgan EN, et al. Inhibition of interleukin-8 blocks myocardial ischemia-reperfusion injury. J Thorac Cardiovasc Surg 1998; 116: 114-21.

49 Boisvert WA, Santiago R, Curtiss LK, Terkeltaub RA. A leukocyte homologue of the IL-8 receptor CXCR-2 mediates the accumulation of macrophages in atherosclerotic lesions of LDL receptor-deficient mice. J Clin Invest 1998; 101: 353-63.

50 Schwartz D, Andalibi A, Chaverri-Almada L, Berliner JA, Kirchgessner $\mathrm{T}$, Fang ZT, et al. Role of the GRO family of chemokines in monocyte adhesion to MM-LDL-stimulated endothelium. J Clin Invest 1994; 94: 1968-73.

51 Weber C, Kraemer S, Drechsler M, Lue H, Koenen RR, Kapurniotu A, et al. Structural determinants of MIF functions in CXCR2-mediated inflammatory and atherogenic leukocyte recruitment. Proc Natl Acad Sci USA 2008; 105: 16278-83.

52 Wong BW, Wong D, McManus BM. Characterization of fractalkine (CX3CL1) and CX3CR1 in human coronary arteries with native atherosclerosis, diabetes mellitus, and transplant vascular disease. Cardiovasc Pathol 2002; 11: 332-8.

53 Lucas AD, Bursill C, Guzik TJ, Sadowski J, Channon KM, Greaves DR. Smooth muscle cells in human atherosclerotic plaques express the 
fractalkine receptor CX3CR1 and undergo chemotaxis to the CX3C chemokine fractalkine (CX3CL1). Circulation 2003; 108: 2498-504.

54 Yang XP, Mattagajasingh S, Su S, Chen G, Cai Z, Fox-Talbot K, et al. Fractalkine upregulates intercellular adhesion molecule-1 in endothelial cells through CX3CR 1 and the Jak Stat5 pathway. Circ Res 2007; 101: 1001-8.

55 Schulz C, Schäfer A, Stolla M, Kerstan S, Lorenz M, von Brühl ML, et al. Chemokine fractalkine mediates leukocyte recruitment to inflammatory endothelial cells in flowing whole blood: a critical role for P-selectin expressed on activated platelets. Circulation 2007; 116: 764-73.

56 Barlic J, Zhang Y, Murphy PM. Atherogenic lipids induce adhesion of human coronary artery smooth muscle cells to macrophages by upregulating chemokine CX3CL1 on smooth muscle cells in a TNFalphaNFkappaB-dependent manner. J Biol Chem 2007; 282:19167-76.

57 White GE, Tan TC, John AE, Whatling C, McPheat WL, Greaves DR. Fractalkine has anti-apoptotic and proliferative effects on human vascular smooth muscle cells via epidermal growth factor receptor signalling. Cardiovasc Res 2010; 85: 825-35.

58 Apostolakis S, Krambovitis E, Vlata Z, Kochiadakis GE, Baritaki S, Spandidos DA. CX3CR1 receptor is up-regulated in monocytes of coronary artery diseased patients: impact of pre-inflammatory stimuli and renin-angiotensin system modulators. Thromb Res 2007; 121: 387-95.

59 Schafer A, Schulz C, Eigenthaler M, Fraccarollo D, Kobsar A, Gawaz $\mathrm{M}$, et al. Novel role of the membrane-bound chemokine fractalkine in platelet activation and adhesion. Blood 2004; 103: 407-12.

60 Combadiere C, Potteaux S, Gao JL, Esposito B, Casanova S, Lee EJ, et al. Decreased atherosclerotic lesion formation in CX3CR1/ apolipoprotein E double knockout mice. Circulation 2003; 107: 1009-16.
61 Saederup N, Chan L, Lira SA, Charo IF. Fractalkine deficiency markedly reduces macrophage accumulation and atherosclerotic lesion formation in CCR2-/- mice: evidence for independent chemokine functions in atherogenesis. Circulation 2008; 117: 1642-8.

62 Landsman L, Bar-On L, Zernecke A, Kim KW, Krauthgamer R, Shagdarsuren $\mathrm{E}$, et al. CX3CR1 is required for monocyte homeostasis and atherogenesis by promoting cell survival. Blood 2009; 113: 963-72.

63 Dorgham K, Ghadiri A, Hermand P, Rodero M, Poupel L, Iga M, et al. An engineered CX3CR1 antagonist endowed with anti-inflammatory activity. J Leukoc Biol 2009; 86: 903-11.

64 Vergunst CE, Gerlag DM, von Moltke L, Karol M, Wyant T, Chi X, et al. MLN3897 plus methotrexate in patients with rheumatoid arthritis: safety, efficacy, pharmacokinetics, and pharmacodynamics of an oral CCR1 antagonist in a phase Ila, double-blind, placebo-controlled, randomized, proof-of-concept study. Arthritis Rheum 2009; 60: 357281.

65 Vergunst CE, Gerlag DM, Lopatinskaya L, Klareskog L, Smith MD, van den Bosch F, et al. Modulation of CCR2 in rheumatoid arthritis: a double-blind, randomized, placebo-controlled clinical trial. Arthritis Rheum 2008; 58: 1931-9.

66 Crawford KW, Li C, Keung A, Su Z, Hughes MD, Greaves W, et al. Pharmacokinetic/pharmacodynamic modeling of the antiretroviral activity of the CCR5 antagonist Vicriviroc in treatment experienced HIVinfected subjects (ACTG protocol 5211). J Acquir Immune Defic Syndr 2010; 53: 598-605.

67 Suleiman J, Zingman BS, Diaz RS, Madruga JV, DeJesus E, Slim J, et al. Vicriviroc in combination therapy with an optimized regimen for treatment-experienced subjects: 48-week results of the VICTOR-E1 phase 2 trial. J Infect Dis 2010 15; 201: 590-9. 